Original Research Paper

\title{
Multi-Objective Evolutionary Optimization of Sandwich Structures: An Evaluation by Elitist Non-dominated Sorting Evolution Strategy
}

\author{
${ }^{1}$ Ilyani Akmar, A.B., ${ }^{2}$ O. Kramer and ${ }^{3}$ T. Rabczuk \\ ${ }^{I}$ Faculty of Civil Engineering, Universiti Teknologi MARA (UiTM), 40450 Shah Alam, Selangor, Malaysia \\ ${ }^{2}$ Department of Computer Science, University of Oldenburg, 26111 Oldenburg, Germany \\ ${ }^{3}$ Institute of Structural Mechanics, Bauhaus University of Weimar, Marienstrasse 15, 99423 Weimar, Germany
}

Article history

Received: 02-01-2015

Revised: $12-02-2015$

Accepted: 12-02-2015

Corresponding Author:

Rabczuk, T./Ilyani Akmar, A.B. Institute of Structural Mechanics,

Bauhaus University of Weimar,

Marienstrasse 15, 99423 Weimar,

Germany

Faculty of Civil Engineering,

MARA University of Technology, 40450 Shah Alam, Selangor,

Malaysia

Email: timon.rabczuk@uni-weimar.de Email: ilyan104@salam.uitm.edu.my

\section{Introduction}

Sandwich-structured composites are a special class of composite materials with the typical features of low weight, high stiffness and high strength. The sandwich structure is fabricated by attaching two thin, strong and stiff skins to a lightweight and relatively thick core made of hybrid materials, Fig. 1 (DIAB, 2012).

The separation of the skins (faces), which actually carry the load, by a low density core, increases the moment of inertia of the panel with little increase in weight producing an efficient structure (Ashby, 2011). The studies on sandwich structures have been extensively continued due to the advantages in sandwich structures. Many researchers have addressed

\begin{abstract}
In this study, an application of evolutionary multi-objective optimization algorithms on the optimization of sandwich structures is presented. The solution strategy is known as Elitist Non-Dominated Sorting Evolution Strategy (ENSES) wherein Evolution Strategies (ES) Genetic algorithm (NSGA-II) procedure. Evolutionary algorithm seems patible approach to resolve multi-objective optimization problems Artificial Intelligence (AI) techniques and elitism cosely linked to important factor for improving evolutionary multi-objective search. In order to evaluate the notion of performance by ENSES, the well-known study case of sandwich structures are reconsidered. For Case 1, the goals and the weight of the sandwich structures. The length, the core and skin hicknesses are the design variables of Case 1. For Case 2, the objective functions are the fabrication cost, the beam weight and the end i.e, the weld height, the weld length, the beam depth and the beam width in Case 2. Numerical results are presented in terms of Pareto-
\end{abstract} Keywords: Multi-objective Evolutionary Optimization, Elitist NonDominated Sorting Evolution Strategy (ENSES), Sandwich Structure, Pareto-Optimal Solutions, Evolutionary Algorithm the experimental, analytical and numerical analysis on sandwich structures such as Rabczuk et al. (2004; Can et al., 2010; Bannister et al., 1999; Guilleminot et al., 2008; Lascoup et al., 2010). The subject interests in sandwich structures are mainly on interfacial fracture, optimization of the designation, delamination, fabrication techniques and influence of changes in sandwich components, i.e., skins and core.

Early applications to multi-objective optimization problems were mainly preference-based techniques although the focus is on finding multiple trade-off solutions (Deb, 2001). Some of the earliest studies are those of Schaffer (1985) who invented in VectorEvaluated Genetic Algorithm (VEGA) and Goldberg (1989) who pioneered in Multi-Objective optimization 
Algorithm (MOEA) through the concept of domination. Through the idea of Goldberg (1989), many researchers expanded the MOEAs with numerous of implementations. As such, Genetic Algorithm (GA) (Fonseca and Fleming, 1993), Non-dominated Sorting GA (NSGA) (Srinivas and Deb, 1994) and niched Pareto-GA (NPGA) (Horn et al., 1994). Later, implementations of EAs in MOEAs are adopted and innovative techniques are developed. For instance, Kursawe's diploidy approach (Kursawe, 1991), Hajela and Lin's weight-based approach (Hajela and Lin, 1992) and Osyczka and Kundu's distance based GA (Osyczka and Kundu, 1995). A comparative study of those techniques is carried out and well-delineated by Zitzler (1999).

Consequently the studies on multi-objective optimization problems have been extensively explored and several methods are proposed to help the decision-maker in the optimization process. For instance, Zitzler et al. (2000; Tan et al., 2001) carried out a comprehensive performance assessment on different multi-objective evolutionary methods. Then, a region-based selection evolutionary multi-objective optimization (PESA-II) by Corne et al. (2001), a fast elitist multi-objective genetic algorithm (NSGA-II) by Deb et al. (2002), a Strength Pareto Evolutionary Algorithm II (SPEA2) by Amuso and Enslin (2007) and improved SPEA2 by Zitzler et al. (2001), an Adapting scatter search to multi-objective optimization (AbYSS) by Nebro et al. (2008) as well as Liu et al. (2010) on a Multiobjective Evolutionary Algorithm based on Decomposition (MOEA/D-DE) using Differential
Evolution (DE). Rangavajhala et al. (2006; Chen et al., 2012) suggested on Robust Design Optimization (RDO) and a new efficient sequential approximate MultiObjective Optimization (MOO) method for by obtaining the Pareto-optimal points respectively. Mosavi and Vaezipour (2012) developed a method on the basis of Reactive Search Optimization (RSO) algorithms in solving engineering optimal design and compared this method with Interactive Multi-objective Optimization and Decision-making method.

An important task in multi-objective optimization is to identify a set of optimal trade-off solutions (called a Pareto set) between the conflicting objectives, which helps gain a better understanding of the problem structure and supports the decision-maker in choosing the best compromise solution for the considered problem. It can be regarded as a population-based stochastic generate-and-test algorithm. It deals simultaneously with a set of possible solutions (socalled population), which allows to find an entire set of Pareto-optimal solutions in a single run of the algorithm. This approach is different to the traditional mathematical programming techniques, which performs a series of separate runs in finding the optimal solution. In addition, evolutionary algorithm is less liable to the shape or continuity of the Pareto-optimal solution, whereas these two factors are the main interest for mathematical programming techniques (Coello, 1999). Elitist Non-dominated Sorting Evolution Strategy (ENSES) by Garcia (2011) is employed to obtain Pareto-optimal solutions.

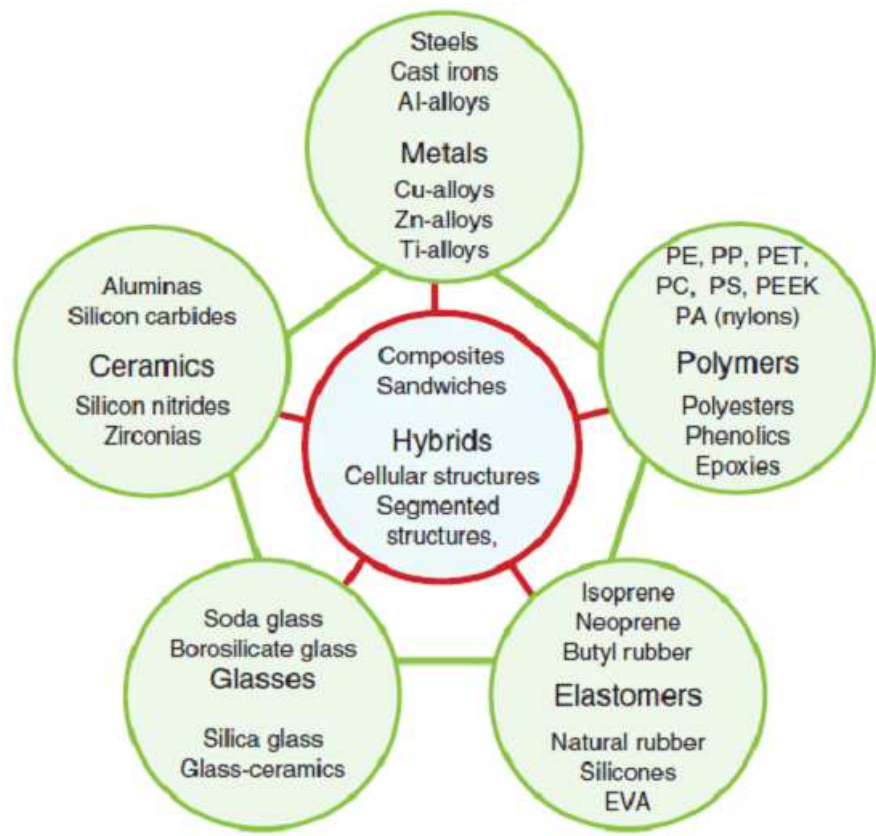

Fig. 1. A chart of hybrid materials that combine two (or more) monolithic materials, or of one material and space. Reproduced by Ashby (2011) 
Fundamentally, ENSES is a sub-division of elitist multi-objective evolutionary algorithms. The ENSES is implemented with an effective sorting method based on individual ranking by non-dominated sorting and a crowded distance metric sorting, which evaluates the population density of solutions in the same rank as defined in NSGA-II. It also has a good search performance for widely distributed Paretooptimal solutions with conflicting objectives. The difference is that this function uses Evolution Strategies (ES) instead of Genetic Algorithm (GA) as Evolutionary Algorithm (EA) in the NSGA-II procedure for multi-objective optimization.

The application adopted in the present paper could serve as the basis comparison for a study of multiobjective optimization with several objective functions. For Case 1, minimization of the deflection and the weight of the sandwich structure are presented. For Case 2 , the fabrication cost of the joint is minimized as well as the end deflection and the weight of the beam structure. The multi-objective optimization formulation is carried out by employing the ENSES to these objective functions. The idea of this work is motivated from the studies of Mosavi and Vaezipour (2012; Steeves and Fleck, 2004a; Garcia, 2011). The rest of the paper is organised as follows. A short resume of ENSES principal evolutionary technique is explained in section 2 followed by the elaboration of sandwich structures in section 3. Section 4 delineates the problem formulations and the optimization criteria. The numerical results and discussions on the notion of performance are realized in section 5 before the paper is concluded in section 6 .

\section{Elitist Non-Dominated Sorting Evolution Strategy (ENSES)}

ENSES is an elitist multi-objective evolutionary algorithm that implements "evolution strategy" as evolutionary algorithm. ES is a variant of Evolutionary Algorithms (EA), which uses mutation, recombination and selection applied to a population of individuals containing candidate solutions in order to evolve iteratively better and better solutions (Beyer and Schwefel, 2002). Evolution strategies were invented by Rechenberg (1973; Schwefel, 1981) and illustrate selfadaptation of strategy parameters in evolutionary computing. The self-adaptivity is defined as that some parameters of EA are varied during a run where the parameters are included in the genes and coevolved with the solutions (Eiben and Smith, 2003). The ENSES algorithm is based on NSGA-II by Deb et al. (2002). Hussain (1998) stated that for an evolution strategy, a fixed-length real-valued vector is utilized to illustrate the representation. This means that each position in the vector corresponds to a feature of the individual.
There are two main reproduction operators in ES; (a) Gaussian mutation: A random value from a Gaussian distribution is added to each element of an individual's vector to create a new offspring. (b) Intermediate recombination: The vectors of two parents are averaged together, element by element, to form a new offspring. These operators reflect the behavioral interpretation of the representation due to the usage of vector element values to derive the new vector elements. The selection of parents to form offspring is less constrained than the parent's selection in genetic algorithms and genetic programming. In common practice of an ES, parents are selected randomly (not based upon fitness) and offspring are generated through the use of recombination. Then, survivors are selected deterministically in which the survivors are chosen either from the best offspring (with no parents survive) or from the best parents and offspring (Spears et al., 1993).

The algorithm of ENSES only works with the ' $\mu+\lambda$ ' selection scheme. This means that ENSES is designed for the minimization problems only. For any maximization problems, we need to modify the original objective for maximization into an objective for minimization by using the 'Duality principle'. This principle can be achieved by multiplying the objective function with -1 . The domination function computes the 'Domination matrix', which specifies the domination relation between two individuals using constraineddomination. The recombination and mutation functions perform the recombination and mutation required in evolution strategies to obtain the next offspring population. There are seven options of recombination offered by ENSES. We adopt discrete recombination for the variables recombination whilst intermediate recombination for the strategy parameters. The nondominated sorting algorithm identifies the different fronts using an $O\left(M N^{2}\right)$ non-dominated sorting procedure, which is taken from section 2.4.7 of Deb (2001). It also carries out the 'Crowded Tournament Selection', so the function returns the parent population of the next generation. The ENSES procedure is outlined in the following (Garcia, 2011):

Step 1: Initialize the population. Problem range and constraints are identified to set the population.

Step 2: Perform ENSES. Evolution strategy, elitism and evaluations are made in this stage. ' $\mu+\lambda$ ' concept is used.

Step 3: Perform fronts and crowded tournament selection operator.

Step 4: Selection. Better results are stored.

Step 5: Create offspring population by using the crowded tournament selection, crossover and mutation operators. Increase generation counter. 
Step 6: Output data. The $\min _{x}$ and $\min _{f}$ are derived, which minimizes the objective function $f$ and achieved minimum of the function $f$, respectively.

\section{Elitist Non-Dominated Sorting Genetic Algorithm (NSGA-II)}

The elitist Non-dominated Sorting Genetic Algorithm (NSGA-II) by Deb et al. (2002) is an elitist multi-objective evolutionary algorithm with time complexity of in generating non-dominated fronts in one generation for population size and objective functions. The crowding distance sorting is an essential part in ranking the population and the best individuals in terms of non-dominance and diversity are then chosen (Cao et al., 2011). It measures on how close an individual is to its neighbours. Large average crowding distance will result in better diversity in the population. In order to define the solutions of the first non-dominated front in a population of size $N$, a domination computation needs to be done where each solution can be compared with every other solution in the population to find if it is dominated. This requires at most $O(M N)$ computations for each solution, where $M$ is the objective function. When this process is continued in order to find all members of the first non-dominated level in the population, thus the overall complexity of the NSGA-II is given by $O\left(M N^{2}\right)$. The NSGA-II procedure is summarized as shown in Fig. 2 (Deb, 2001; Seshadri, 2011).

\section{Sandwich Composite Structures}

Sandwich structures with composite skin sheets that combine high stiffness, strength and mechanical energy absorption with low weight are widely used in the aerospace, marine, automobile, locomotive, buildings and consumer industries (Xia and $\mathrm{Wu}, 2010$; Mori et al., 2007). A typical sandwich composite structure is used to analyze the multi-objective optimization as depicted in Fig. 3.

The skins carry most of the load (tensile and compressive loads) in the sandwich structures and the local flexural rigidity is negligible (extremely small value). The skins form the exterior surfaces and withstand the shear forces when the local pressure is high. Commonly, fibre reinforced composites are used as the skin materials. The separation of the skins by the core increases the moment of inertia of the section and its section modulus producing a structure that resists bending and buckling loads well. Correspondingly, the core has to be stiff enough to ensure the skins do not slip over each other and make the sandwich structure behave as a load-bearing unit
(Ashby, 2011). An adhesive layer also provided in a sandwich structure to assure the core and skins are bonded together. Adhesive layers will carry the shear and tensile stresses as same as the shear stress in core (DIAB, 2012).

There are several reasons for the usage of sandwich composites structures;

\section{Decrease Weight}

They provide mechanical properties to much lower weight than traditional monolithic materials such as steels. For instance, in transportation, this property gives a robust impact wherein a lower weight in vessel construction enables higher payloads resulting in reduced emissions.

\section{Environmentally Friendly}

Since the usage of sandwich composites will make a lighter structural design thus less material is consumed in the construction consequently less energy-consuming over its lifetime.

\section{Free Design}

Deformability in sandwich composites gives an advantage in designing stage. This allows non-linear and smooth designs not only for the aesthetic and aerodynamic reasons.

\section{Thermal Insulation}

Great impact to sub-sea application due to the cell structure in core materials, which filled with air. This prevents the heat or cold transfusion.

\section{Sound Insulation}

The core material has the ability to absorb sound.

\section{Corrosion Resistance}

The non-corrosive properties of sandwich structures prevent the risk of corrosion attack on structures. Suitable for marine structures.

\section{Very Low Water Absorption}

The closed cells in sandwich structures prevent moisture from penetrating the core consequently increasing the weight and destroy the structures.

\section{Repairing Aspect}

Cracks can be repaired without any major work as compared to steel thus will not affect the performance of the structures.

It is noted that the sandwich beams prone to fail by one of several potential failure modes, Fig. 4 (Ratwani, 2010; Composites, 2000; Steeves and Fleck, $2004 b$ ); the operative mode is defined by the material 
properties, beam geometry and the loading configuration. Four failure modes which regularly arise in sandwich beams are core shear $P_{C S}$, skin yielding or microbuckling $P_{M B}$, ductile indentation $P_{D I}$ and elastic indentation $P_{E I}$. Core shear failure is caused by insufficient core shear strength or panel thickness. It also occurs when the shear strength of the core is exceeded. The maximum shear strength $P_{C S}$ can be estimated by Equation 1:

$$
P_{C S}=2 b\left(t_{f}+t_{c}\right) \tau_{c}
$$

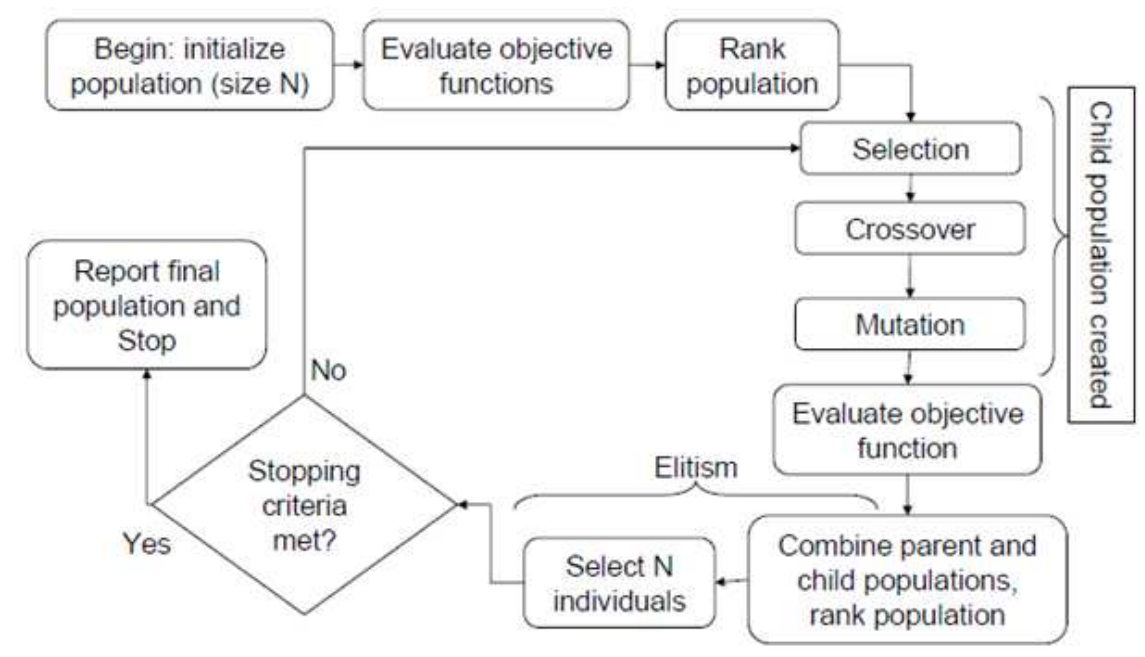

Fig. 2. A flowchart of NSGA-II. Reproduced by Deb and Goel (2001)

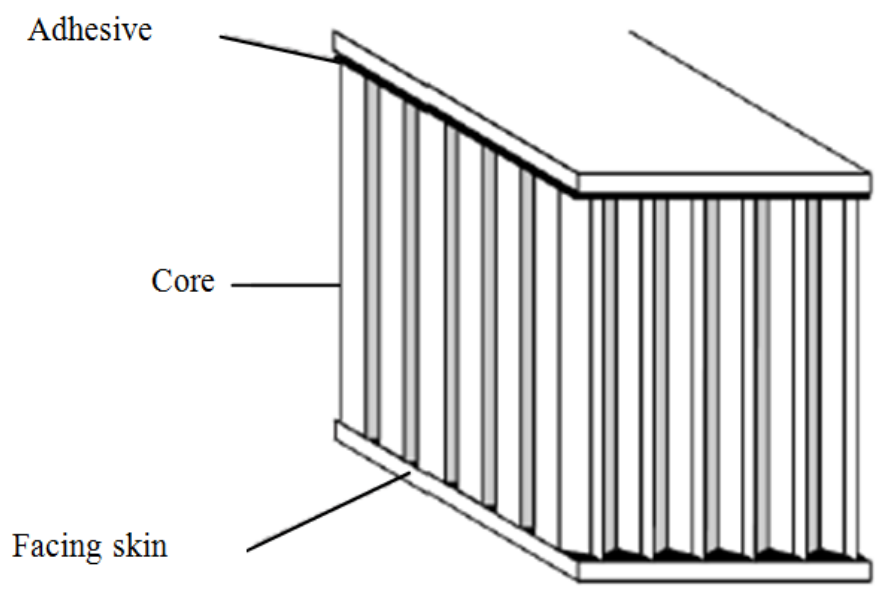

Fig. 3. A typical sandwich composite structure. Reproduced by Composites (2000)

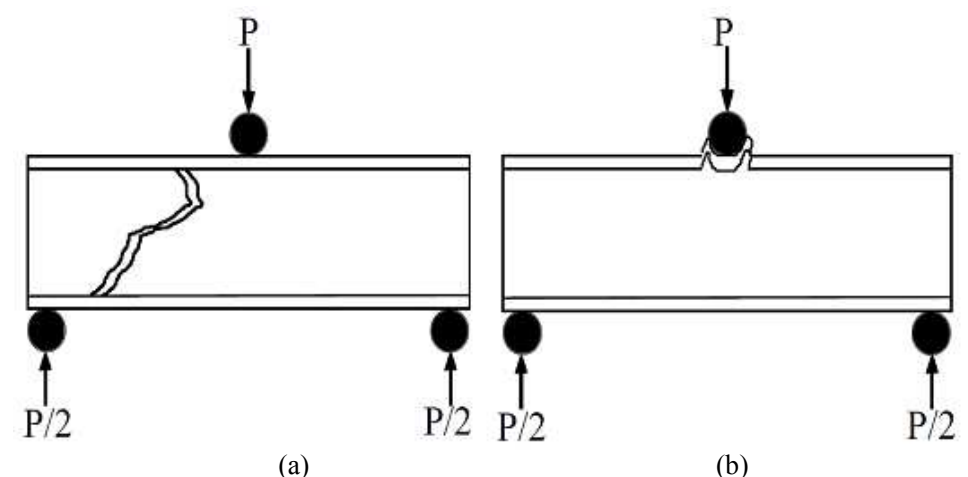




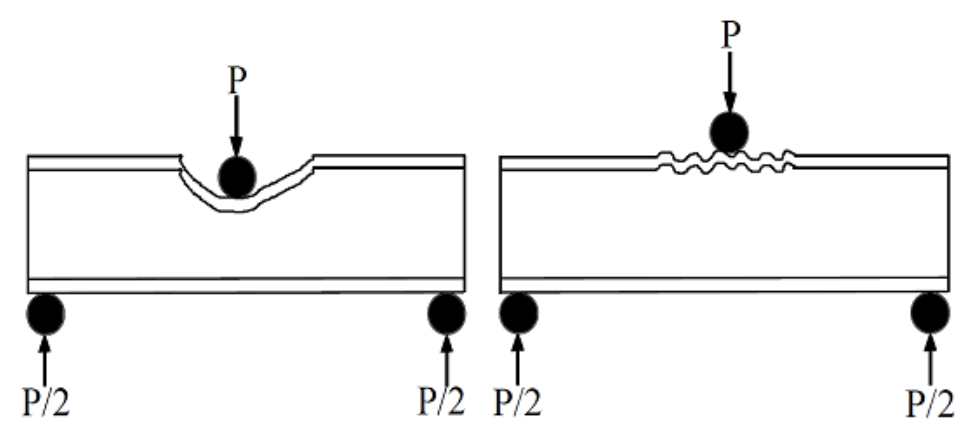

(c) (d)

Fig. 4. Failure modes in sandwich beam structures. Reproduced by Steeves and Fleck (2004b) (a) Core shear (b) Microbuckling (c) Indentation (d) Face wrinkling

Skin yielding or microbuckling occurs when the axial stress in the sandwich skin attains the yield or microbuckling strength $P_{M B}$ of the skin material given by Equation 2:

$P_{M B}=\frac{4 b\left(t_{f}+t_{c}\right) \sigma_{f}}{L}$

The expression for the maximum load $P D I$ in ductile indentation problem is as Equation 3:

$P_{D I}=2 b t_{f}\left(\sigma_{c} \sigma_{f}\right)^{1 / 2}$

The maximum indentation load $P E I$ referring to an elastic indentation failure can be computed by Equation 4:

$P_{E I}=b t_{f}\left(\frac{\pi^{2}\left(t_{f}+t_{c}\right) E_{f} \sigma_{c}^{2}}{3 L}\right)^{1 / 3}$

Conclusively, a sandwich structure is designed to ascertain that it is capable to withstand the structural loads throughout its design life and its structural integrity during the in-service environments.

\section{Problem Formulation}

The two optimization tasks are formulated as follows.

\section{Case 1: A Simply Supported Sandwich Beam}

A sandwich beam as illustrated in Fig. 5 is adopted where the span of the beam is $l_{s}$, the width $b$, the core thickness $t_{c}$ and the skin thickness, $t_{f}$. Assume a 3-point loading is imposed to the beam. However, the analysis is identical for other forms of bending load with the exception of the different values for the beam geometrical constants $k$. When subject to a load $P$, the beam is prone to deflect. The deflection of a sandwich beam $\delta$ is the sum of the bending deflection $\delta_{\mathrm{b}}$ and the shear deflection $\delta_{\mathrm{s}}$. The bending deflection $\delta_{\mathrm{b}}$ depends on the relative tensile and compressive moduli of the skin materials whilst the shear deflection $\delta_{s}$ is dependent on the shear modulus of the core. The stiffness of the beam in bending is calculated from the equivalent flexural rigidity $(E I)_{e q}$ and the equivalent shear rigidity $(A G)_{e q}$ by Equation 5:

$(E I)_{e q}=\frac{E_{f} b t_{f} h_{b}^{2}}{2}$
$(A G)_{e q}=b h_{b} G_{c}$

where, $E_{f}$ and $G_{c}$ are the skin's modulus of elasticity and core shear modulus (in direction of the applied load), respectively and $h_{b}=t_{f}+t_{c}$ is the distance between facing skin centres. The deflection of the beam $\delta$ can be written as Equation 6:

$\delta=\frac{k_{b} P l_{s}^{3}}{(E I)_{e q}}+\frac{k_{s} P l_{s}}{(A G)_{e q}}$

where, $k_{b}$ and $k_{s}$ are the bending and shear deflection coefficients respectively as summarized in Table 1 . The $\delta$ value is the objective function that needs to be minimized. The other key issue in designing sandwich beams is the minimization of weight. The weight of the beam $W$ is governed by Equation 7:

$W=2 \rho_{f} g b l_{s} t_{f}+\rho_{c} g b l_{s} t_{c}$

where, $g$ is the acceleration due to gravity. This problem formulation leads to two nonlinear objective functions with respect to five nonlinear inequality constraints. The length $l_{s}$, the core $t_{c}$ and skin thicknesses $t_{f}$ are the design variables of Case 1 . 
Table 1. Beam geometrical constants for different types of loading modes. Reproduced by Composites (2000)

\begin{tabular}{|c|c|c|c|c|}
\hline Beam type & $\begin{array}{l}\text { Maximum shear } \\
\text { force } F\end{array}$ & $\begin{array}{l}\text { Maximum bending } \\
\text { moment } \mathrm{M}\end{array}$ & $\begin{array}{l}\text { Bending deflection } \\
\text { coefficient } \mathrm{k}_{\mathrm{b}}\end{array}$ & $\begin{array}{l}\text { Shear deflection } \\
\text { coefficient } \mathrm{k}_{\mathrm{s}}\end{array}$ \\
\hline \multirow{2}{*}{ Simply Supported with Uniform Distributed Load (SS-UDL) } & $P$ & $\mathrm{Pl}$ & 5 & 1 \\
\hline & $\overline{2}$ & $\overline{8}$ & $\overline{384}$ & $\overline{8}$ \\
\hline \multirow{2}{*}{ Fixed-Fixed end with Uniform Distributed Load (FF-UDL) } & $P$ & $P l$ & 1 & 1 \\
\hline & $\overline{2}$ & $\overline{12}$ & $\overline{384}$ & $\overline{8}$ \\
\hline \multirow{2}{*}{ Simply Supported beam with Central Load (SS-CL) } & $P$ & $P l$ & 1 & 1 \\
\hline & $\overline{2}$ & $\overline{4}$ & $\overline{48}$ & $\overline{4}$ \\
\hline \multirow{2}{*}{ Fixed-Fixed end with Central Load (FF-CL) } & $P$ & $P l$ & 1 & 1 \\
\hline & $\overline{2}$ & $\overline{8}$ & $\overline{192}$ & $\overline{4}$ \\
\hline \multirow{2}{*}{ Cantilever beam with Uniform Distributed Load (Can-UDL) } & \multirow{2}{*}{$P$} & $P l$ & 1 & 1 \\
\hline & & $\overline{2}$ & $\overline{8}$ & $\overline{2}$ \\
\hline Cantilever beam with End Load (Can-EL) & $P$ & $P l$ & $\frac{1}{3}$ & 1 \\
\hline \multirow{2}{*}{ Cantilever beam with Triangular Load (Can-TriL) } & \multirow{2}{*}{$P$} & $P l$ & 1 & 1 \\
\hline & & $\overline{3}$ & $\overline{15}$ & $\overline{3}$ \\
\hline
\end{tabular}

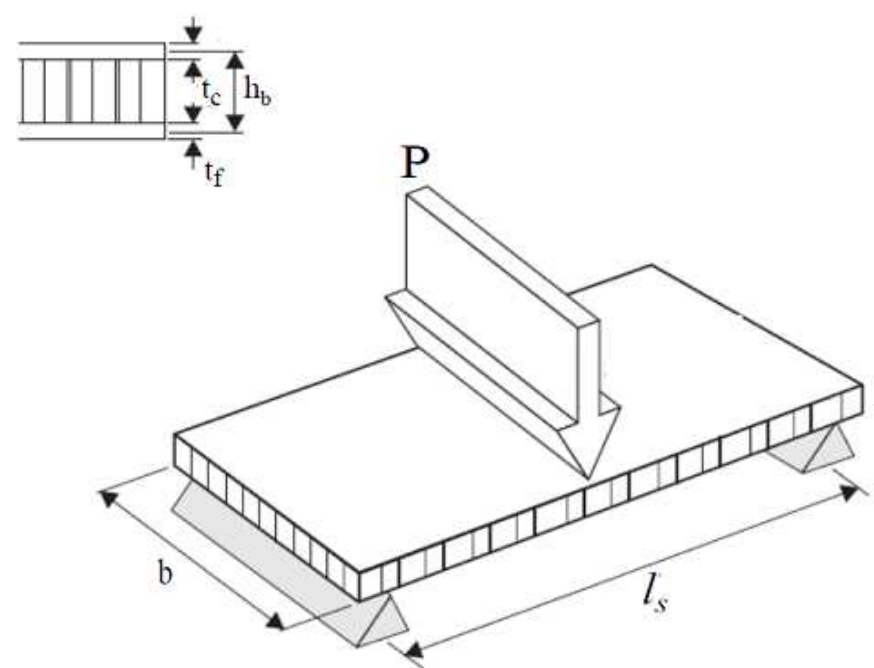

Fig. 5. The geometry configurations of the sandwich beam

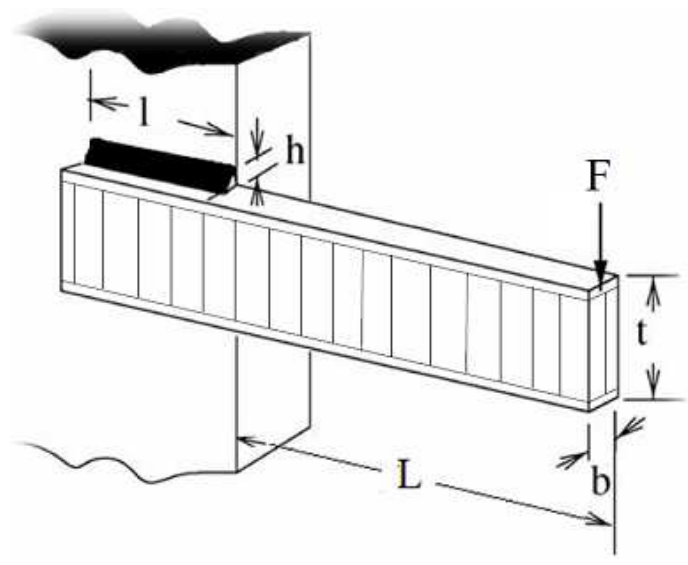

Fig. 6. The welded sandwich beam optimal design problem

A welded sandwich beam design in Fig. 6 is an example that deals with hybrid materials and connecting them to form a complex structure. The beam is welded on another beam carrying a point load $F$. The problem of designing an optimal welded sandwich beam consists of dimensioning a welded sandwich beam and the welding length in order to minimize the fabrication cost $C$, the end deflection of the beam $\delta(x)$ and the beam weight $W$ subjected to bending stress $\sigma_{d}$, constraints on shear stress $\tau_{d}$, the buckling load on the bar $P_{b}$ and side constraints. This problem formulation leads to three nonlinear objective functions with respect to three nonlinear and one linear inequality constraints. The objective is to reduce the fabrication cost of the joint without causing a high deflection that occurs at the beam end as well as the beam weight. Four variables have been identified, i.e. the beam depth $t$, the beam width $b$, the weld length $l$ and the weld thickness $h$. The beam weight expression is similar to the one adopted in Case 1 but with different input variables. The cost $C$ and deflection $\delta(x)$ formulations are governed by Equation 8: 


$$
\begin{aligned}
& C=1.10471 h^{2} l+0.04811 t b(L+l) \\
& \delta(x)=\frac{2.1952}{t b^{3}}
\end{aligned}
$$

The formulation of the four constraints are related to the shear stress $\tau(x)$ developed at the support location of the sandwich beam, which is set to be smaller than the design shear stress $\tau_{d}$. The normal stress $\sigma(x)$ developed at the support location of the sandwich beam is meant to be smaller than the design normal stress value $\sigma_{d}$. The width of the welded sandwich beam $b$ must be greater than the weld thickness $h$ from the standpoint. The beam buckling load $P_{b}$ guarantees a safe sandwich beam design by providing a greater value than the $F$. The constraint expressions are given as follows Equation 9:

$$
\begin{aligned}
& \sigma(x)=\frac{6 F L}{b t^{2}} \\
& \tau(x)=\left(\frac{F}{\sqrt{2} h l}\right)^{2}+\frac{2 l\left(\frac{F}{\sqrt{2} h l}\right)\left(\frac{F[L+l / 2] \sqrt{\frac{l^{2}}{4}+\frac{(t+h)^{2}}{4}}}{\left[2\left(0.707 h l\left(\frac{l^{2}}{12}+\frac{(t+h)^{2}}{4}\right)\right]\right.}\right)}{2\left(\sqrt{\left.\frac{l^{2}}{4}+\frac{(t+h)^{2}}{4}\right)}\right.} \\
& \left.+\left(\frac{\left.F[L+l / 2] \sqrt{\frac{l^{2}}{4}+\frac{(t+h)^{2}}{4}}\right)^{2}}{2\left[0.707 h l\left(\frac{l^{2}}{12}+\frac{(t+h)^{2}}{4}\right)\right]}\right]\right)^{\left[.013 \sqrt{\frac{E_{f} b t_{f} t_{c}^{2}}{2}\left(\frac{1}{3}\left(12 \times 10^{6}\right) t b^{3}\right)}\right.}\left[1-\frac{t}{2 L} \sqrt{\left.\frac{\left(\frac{E_{f} b t_{f} t_{c}^{2}}{2}\right)}{\left(\frac{1}{3}\left(12 \times 10^{6}\right) t b^{3}\right.}\right)}\right] \\
& \left.P_{b}=\frac{(9)}{L^{2}}\right]
\end{aligned}
$$

\section{Optimization Criteria}

We have seen in the previous section that the two optimization tasks result in multi-objective optimization problems. With all of the aforementioned considerations, the two multi-objective function problems are formulated as follows;

\section{A Sandwich Beam:}

$$
\begin{aligned}
& \text { Minimize } f_{1}(x)=W=2 \rho_{f} g b l_{s} t_{f}+\rho_{c} g b l_{s} t_{c} \\
& \text { Minimize } f_{2}(x)=\delta=\frac{2 k_{b} P l_{s}^{3}}{E_{f} b t_{f} h_{b}^{2}}+\frac{k_{s} P l_{s}}{b h_{b} G_{c}}
\end{aligned}
$$

Subject to:

$$
\begin{aligned}
& \mathrm{g}_{1}(x)=\mathrm{P}_{\mathrm{CS}}-\mathrm{P} \geq 0, \\
& \mathrm{~g}_{2}(x)=\mathrm{P}_{\mathrm{MB}}-\mathrm{P} \geq 0, \\
& \mathrm{~g}_{3}(x)=\mathrm{P}_{\mathrm{DI}}-\mathrm{P} \geq 0, \\
& \mathrm{~g}_{4}(x)=\mathrm{P}_{\mathrm{EI}}-\mathrm{P} \geq 0, \\
& \delta \leq \delta_{\max }, \\
& 100 \mathrm{~mm} \leq l \leq 1000 \mathrm{~mm} \\
& 5 \mathrm{~mm} \leq t_{f} \leq 20 \mathrm{~mm} \\
& 10 \mathrm{~mm} \leq t_{c} \leq 50 \mathrm{~mm}
\end{aligned}
$$

\section{A Welded Sandwich Beam Design}

$$
\begin{aligned}
& \text { Minimize } f_{1}(x)=C=1.10471 h^{2} l+0.04811 t b(L+l) \\
& \text { Minimize } f_{2}(x)=\delta(x)=\frac{2.1952}{t b^{3}} \\
& \text { Minimize } f_{3}(x)=W=2 \rho_{f} g b(L+l) t_{f}+\rho_{c} g b(L+l)\left(t-2 t_{f}\right)
\end{aligned}
$$

Subject to:

$$
\begin{aligned}
& g_{1}(x)=\tau_{d}-t(x) \geq 0, \\
& g_{2}(x)=\sigma_{d}-s(x) \geq 0, \\
& g_{3}(x)=\mathrm{P}_{\mathrm{b}}(x)-F \geq 0, \\
& g_{4}(x)=b-h \geq 0, \\
& 3 \mathrm{~mm} \leq h, b \leq 150 \mathrm{~mm} \\
& 3 \mathrm{~mm} \leq l, t \leq 250 \mathrm{~mm}
\end{aligned}
$$

The relevant material properties of the facing skins and the core used in both cases are given in Table 2 .

\section{Simulation Results and Evaluation of Performance}

Evaluating an optimization technique frequently includes the notion of performance. In the case of multiobjective optimization, the quality verification is certainly more complicated than the single-objective optimization problems, due to the multiple optimization goals. Obviously, the goals consist of minimizing the distance of the resulting non-dominated set to the Paretooptimal solution, finding a good distribution of the solutions where the evaluation might be upon the distance metric and maximizing the extent of the obtained non-dominated front wherein a wide range of values should be covered by the non-dominated solutions. In this section, a detailed discussion on results of two optimization problems is presented. For the simulation results, the set of best compromises are given in the form of the associated Pareto-optimal solution.

ENSES performs an elitist multi-objective evolutionary algorithm and the major concern of this technique is avoiding the premature convergence; i.e. converges to a point that is only a local minimiser of the function. In addition, the NSGA-II algorithm used in ENSES for multi-objective problems might return fully 
non-dominated solutions, but non-dominance is not a guarantee for convergence to the Pareto front. This is particularly true for a small population but with a large population of sample size thus acquiring more function evaluations. For multi-objective optimization, convergence of an algorithm occurs simply when all members of that population are non-dominated. In general, ENSES is able to find a good approximation to the problem's global minimum or Pareto front, with high probability. It also indicates other promising regions in the search space, or function as a simple test algorithm to find potential problems with the objective functions. In terms of output presentations, ENSES gives a visualization on the initial generation gen $=0$ and the Pareto optimal front for every runs as depicted in Fig. 7.
According to Fig. $7 \mathrm{a}$ and $\mathrm{b}$ the blue points represent the initialization of the population by random means while the red points are the final solutions produced by the last generation. Through the iterations, the improvement in the solutions becomes smaller and smaller converging against the optimal Pareto set. Looking into the influence of the population size, several runs were performed in order to investigate the influence of the population size as well as the maximum number of generations converging towards the Pareto-optimal front. By taking Case 1 as the sample problem, we could visualize the outcomes (initial generation and Pareto-optimal solutions) of multiple runs with several population sizes by ENSES as in Fig. 8 and 9, respectively.

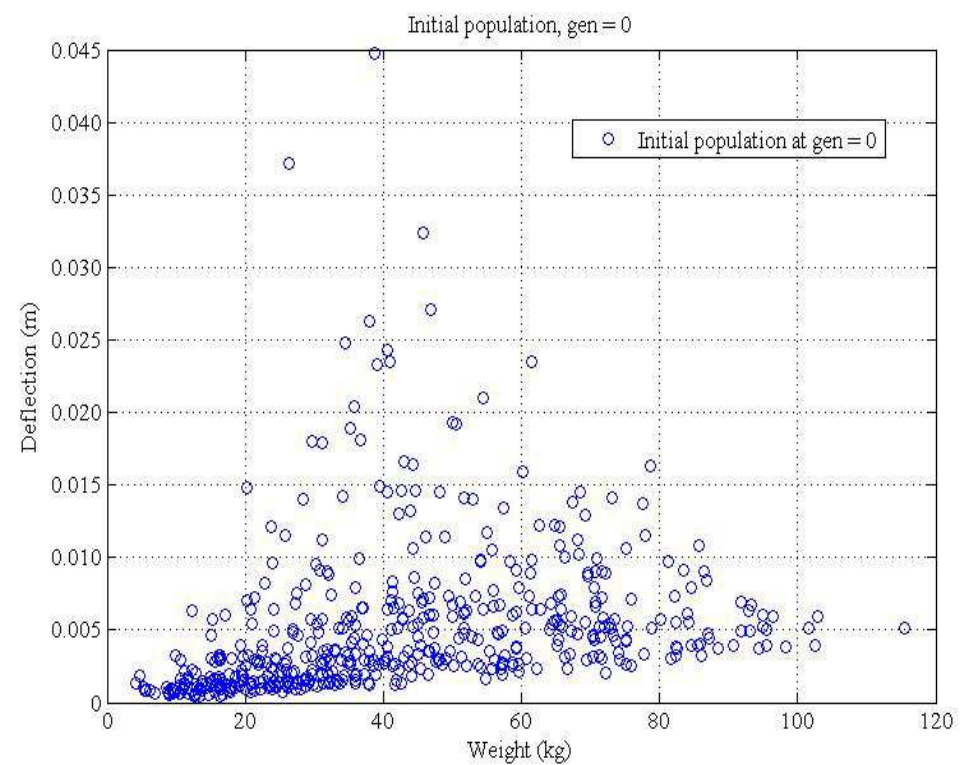

(a)

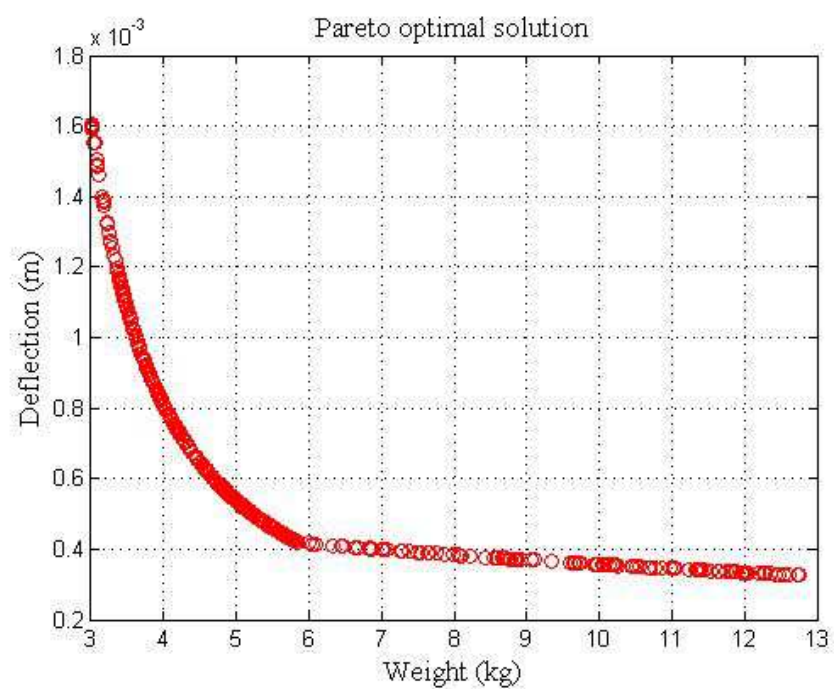

(b)

Fig. 7. Initial generations and Pareto-optimal front in ENSES for simply supported sandwich beam with central load (a) Initial generation visualization (b) Pareto-optimal front visualization 


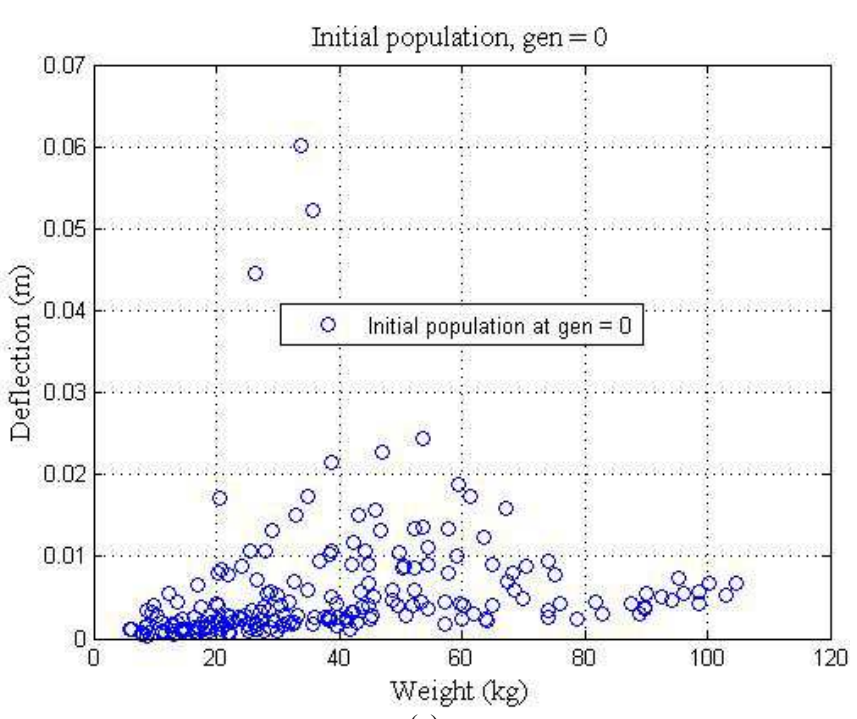

(a)

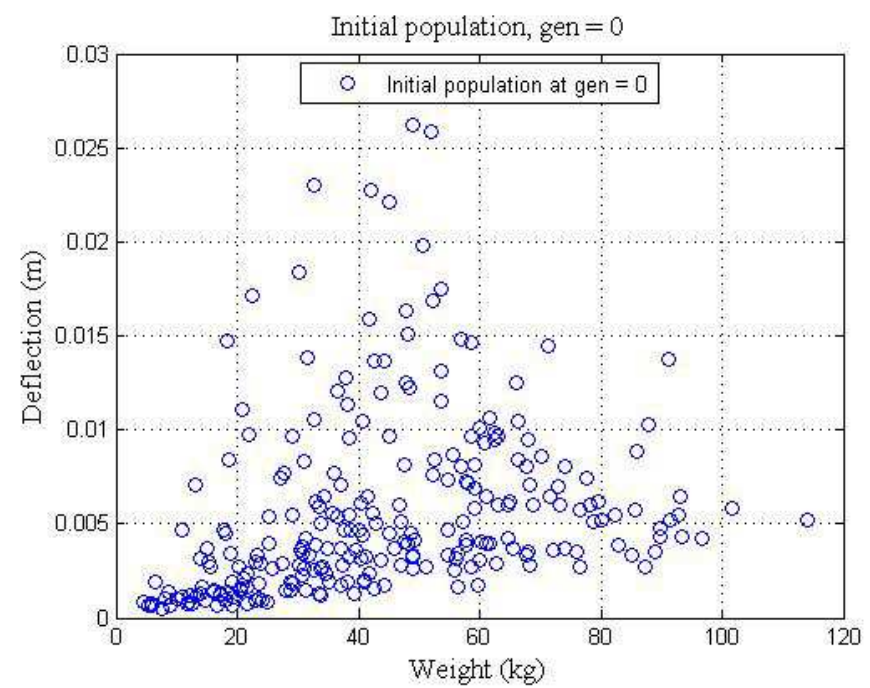

(b)

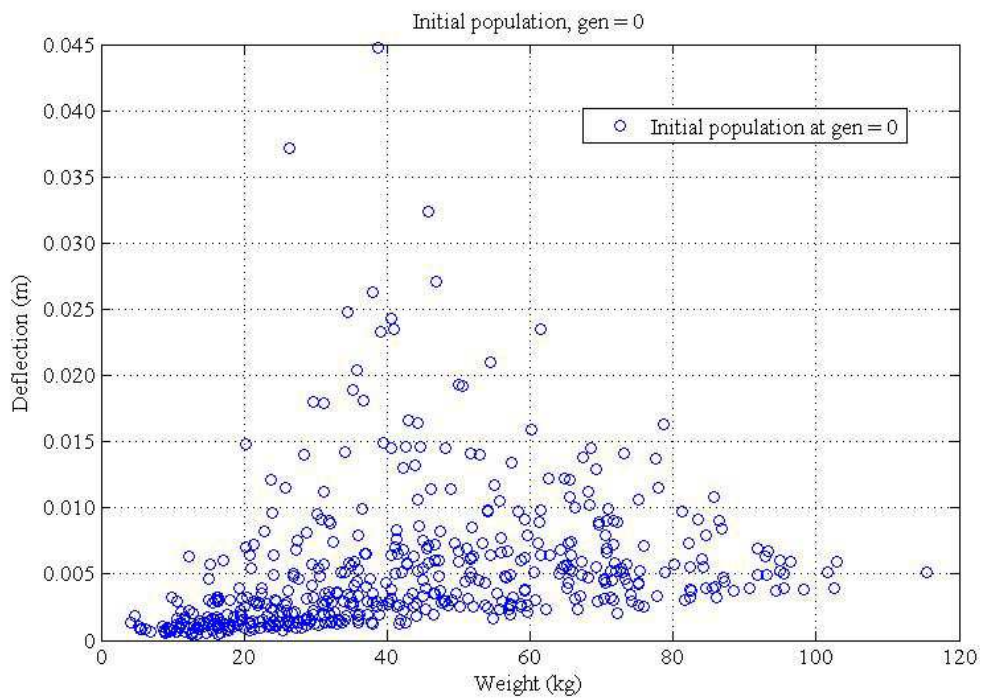

(c) 


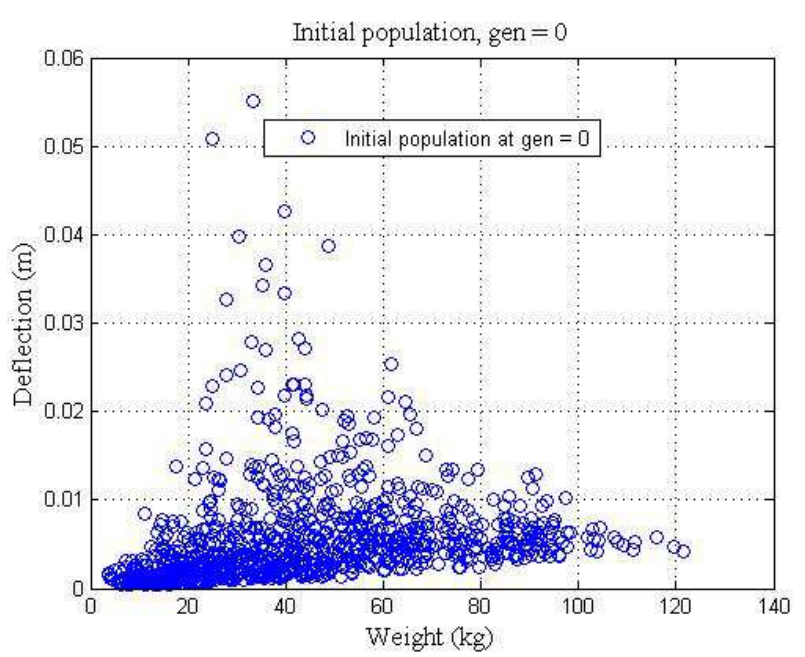

(d)

Fig. 8. Initial generation due to the influence of population sizes and number of generations in Case 1 analysis (a) Initial generation of 200 samples (b) Initial generation of 250 samples (c) Initial generation of 500 samples (d) Initial generation of 1000 samples

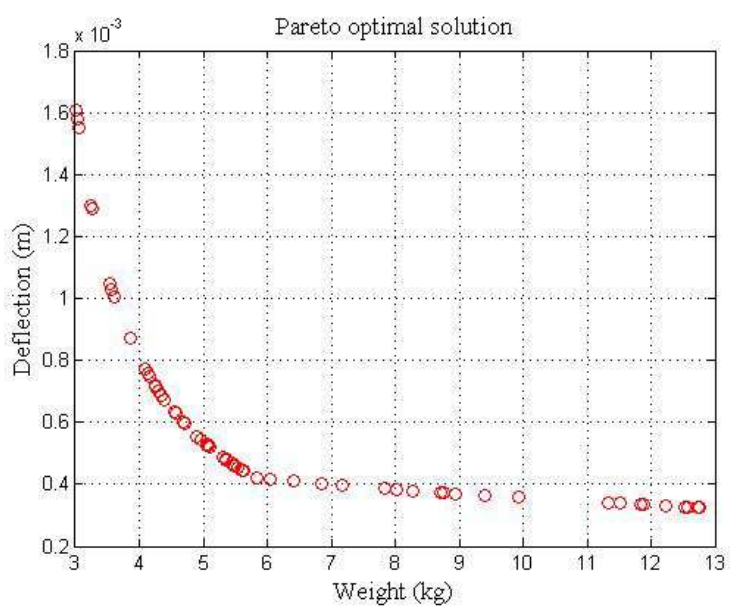

(a)

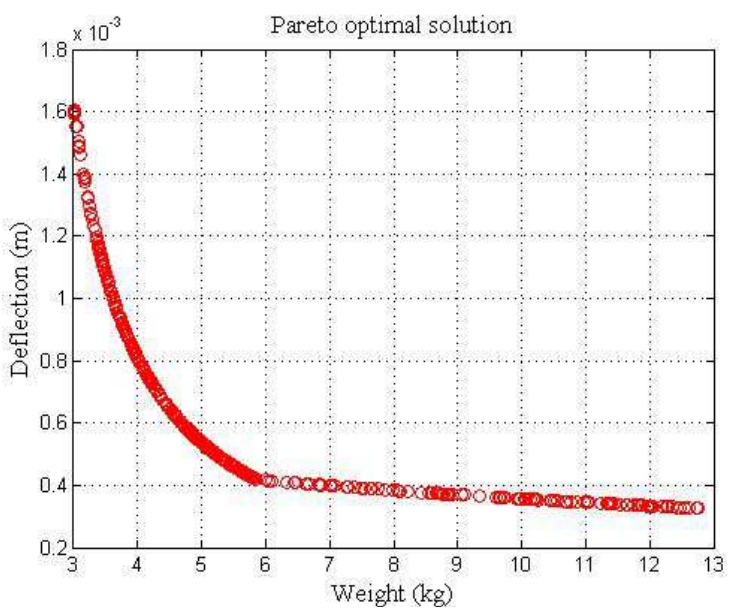

(c)

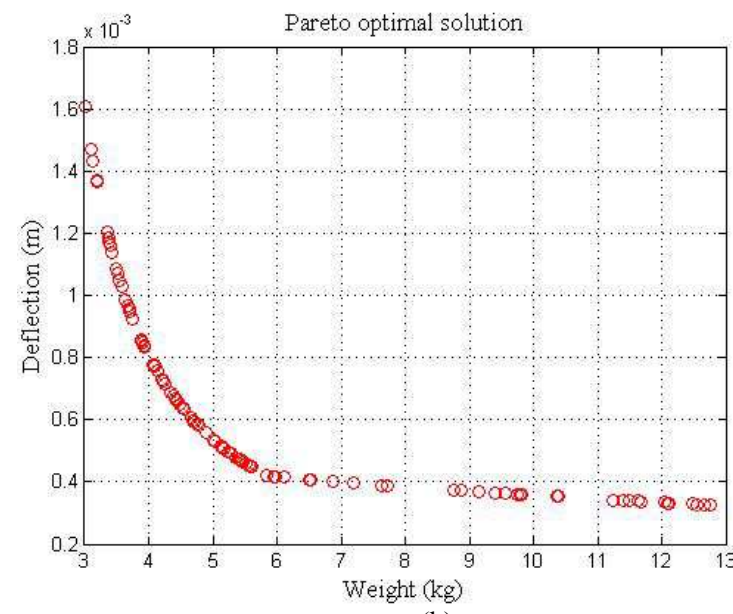

(b)

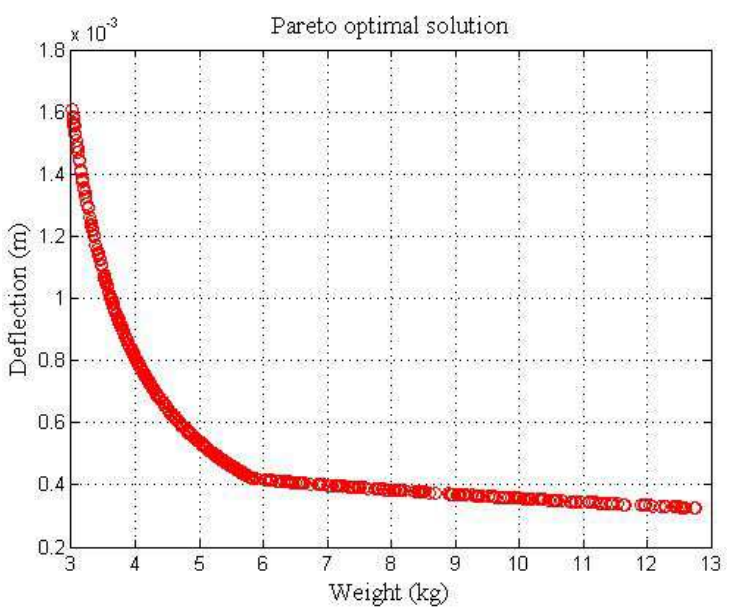

(d)

Fig. 9. Pareto-optimal solutions due to the influence of population sizes and number of generations in Case 1 analysis (a) Paretooptimal solutions of 200 samples (b) Pareto-optimal solutions of 250 samples (c) Pareto-optimal solutions of 500 samples (d) Pareto-optimal solutions of 1000 samples 


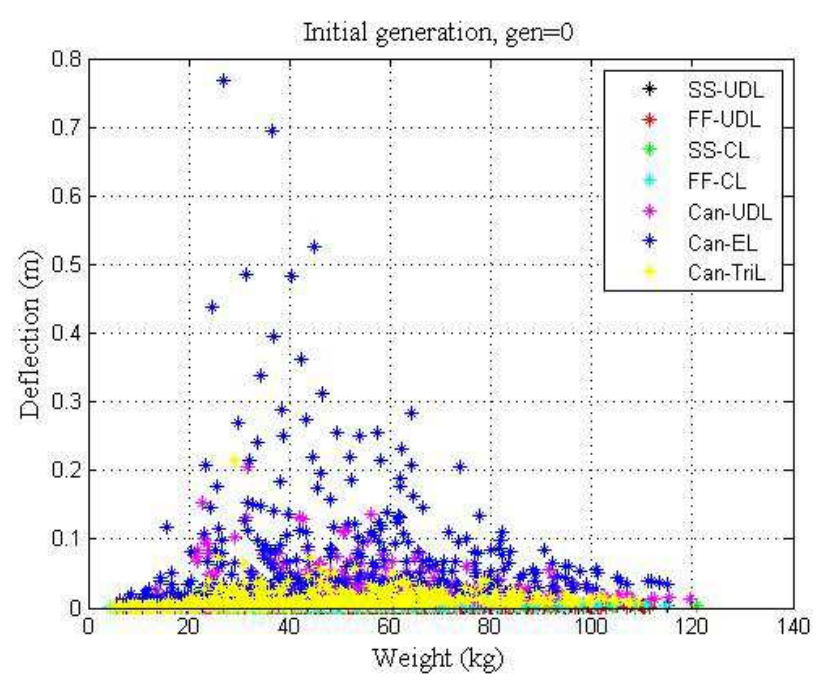

(a)

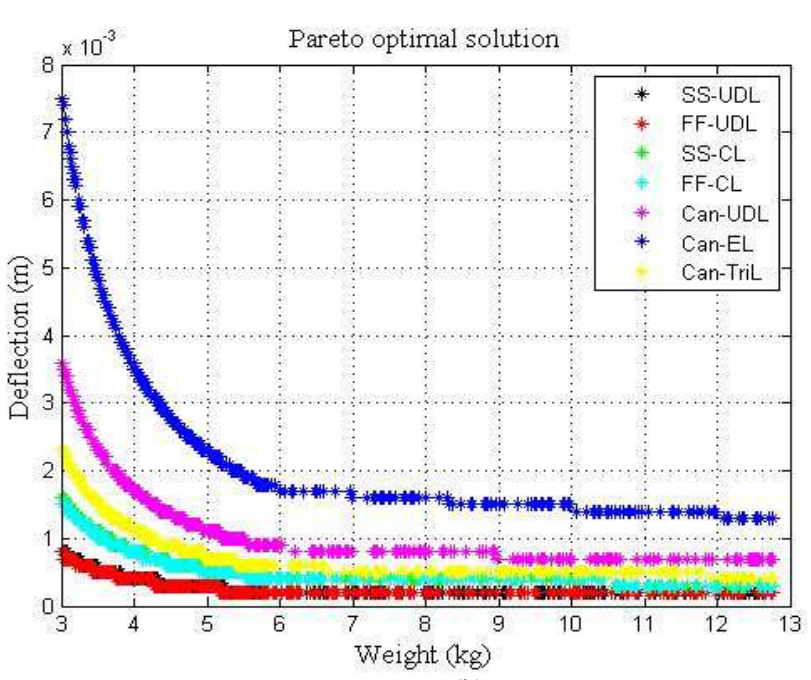

(b)

Fig. 10. Beam evaluations with respect to the boundary conditions: Simply supported with uniform distributed load (SS-UDL); Fixed-Fixed end with Uniform Distributed Load (FF-UDL); Simply supported beam with central load (SS-CL); Fixed-Fixed end with Central Load (FF-CL); Cantilever beam with Uniform Distributed Load (Can-UDL); Cantilever beam with End Load (Can-EL); Cantilever beam with Triangular Load (Can-TriL) (a) Initial generation with different boundary conditions (b) Pareto-optimal solutions with different boundary conditions

Table 2. Sandwich composite configuration and data (Composites, 2000)

\begin{tabular}{|c|c|c|c|c|}
\hline \multirow{2}{*}{$\begin{array}{l}\text { Configuration } \\
\text { Facing skins: }\end{array}$} & \multicolumn{4}{|l|}{ Data } \\
\hline & $\mathrm{S} 1$ & $\mathrm{~S} 2$ & S3 & S4 \\
\hline Compressive strength, $\sigma_{\mathrm{f}}(\mathrm{MPa})$ & 550.0 & 150.0 & 1300.0 & 35.0 \\
\hline Young's modulus, $\mathrm{E}_{\mathrm{f}}(\mathrm{GPa})$ & 20.0 & 70.0 & 115.0 & 9.0 \\
\hline Density, $\rho_{\mathrm{f}}\left(\mathrm{kg} / \mathrm{m}^{3}\right)$ & 470.0 & 1350.0 & 190.0 & 6300.0 \\
\hline Core: & $\mathrm{C} 1$ & $\mathrm{C} 2$ & $\mathrm{C} 3$ & $\mathrm{C} 4$ \\
\hline Compressive strength, $\sigma_{\mathrm{c}}(\mathrm{MPa})$ & 0.9 & 0.4 & 10.0 & 2.9 \\
\hline Core shear strength, $\tau_{\mathrm{c}}(\mathrm{MPa})$ & 15.0 & 1.9 & 4.7 & 1.7 \\
\hline Young's modulus, $\mathrm{E}_{\mathrm{c}}(\mathrm{MPa})$ & 165.0 & 55.0 & 2345.0 & 364.0 \\
\hline Shear modulus, $\mathrm{G}_{\mathrm{c}}(\mathrm{MPa})$ & 600.0 & 55.0 & 1275.0 & 193.0 \\
\hline Density, $\rho_{\mathrm{c}}\left(\mathrm{kg} / \mathrm{m}^{3}\right)$ & 29.0 & 127.0 & 144.0 & 72.0 \\
\hline \multicolumn{5}{|l|}{ Case 1} \\
\hline Applied load, $\mathrm{P}(\mathrm{kN})$ & \multicolumn{4}{|c|}{25.00000} \\
\hline UDL, q (kN/m length) & \multicolumn{4}{|c|}{20.00000} \\
\hline Gravity speed, $g\left(\mathrm{~m} / \mathrm{s}^{2}\right)$ & \multicolumn{4}{|c|}{9.81000} \\
\hline Width, b (m) & \multicolumn{4}{|c|}{0.50000} \\
\hline Max. Deflection, $\delta_{\max }(\mathrm{mm})$ & \multicolumn{4}{|c|}{0.05000} \\
\hline Parent population size, $\mu$ & \multicolumn{4}{|c|}{500.00000} \\
\hline Offspring population size, $\lambda$ & \multicolumn{4}{|c|}{500.00000} \\
\hline No. of generation & \multicolumn{4}{|c|}{500.00000} \\
\hline \multicolumn{5}{|l|}{ Case 2} \\
\hline Point load, F (kN) & \multicolumn{4}{|c|}{25.00000} \\
\hline Overhang beam length, L (m) & \multicolumn{4}{|c|}{0.50000} \\
\hline Skin thickness, $\mathrm{t}_{\mathrm{f}}(\mathrm{m})$ & \multicolumn{4}{|c|}{0.00025} \\
\hline Parent population size, $\mu$ & \multicolumn{4}{|c|}{500.00000} \\
\hline Offspring population size, $\lambda$ & \multicolumn{4}{|c|}{500.00000} \\
\hline No. of generation & \multicolumn{4}{|c|}{500.00000} \\
\hline
\end{tabular}

Figure 10 and 11 describe the resulting Paretofronts with respect to the aforementioned variables from the perspectives of boundary conditions and material properties evaluated by ENSES. Initially the 
beam is assumed to be a simply-supported beam. However, the analysis is identical for other forms of bending load with relevant value of $k$-constants. The ENSES evaluations are executed over 500 generations to optimize the two objectives subjected to the constraints on sandwich beam failure modes and maximum deflection. It can be concluded that an identical result is retrieved between the simplysupported and fixed-fixed end supports with uniform distributed load.

The difference of beam deflection value becomes smaller and smaller as it reaches $7 \mathrm{~kg}$ for SS-UDL, FF-UDL, FF-CL, Can-TriL cases followed by CanUDL at $9 \mathrm{~kg}$ weight. Commonly, a stiff facing skin is attached to a lightweight core. The principle of chosen material for facing skins and core varies from the lowest to the greatest strength as in Table 2. Figure 11 illustrates the heavier the material used in the sandwich beam, the lesser deflection is occurred provided that the same load and boundary condition are applied.

\section{Case 2: Welded Sandwich Beam}

Figure 12 illustrates that along with the step-bystep iterations, the Pareto-optimal solutions become better and better with respect to the overall optimization as well as achieving a greater and greater spread across the three objectives. The interactions of the Pareto-optimal solutions are clearly defined, when we present them in any two of these objectives in two dimensions plotting. The Pareto-optimal solutions reflect extremely well behaved across all three objectives when these are considered in pairs, Fig.
$12 \mathrm{~d}-\mathrm{f}$ wherein the solutions are improving through the iterations and indicating a convergence of the compromise process. The crossing phenomenon due to the action of the various operators, which occurs among these different generations is obvious. This can be seen from the differences between the visualizations of the two-objective optimization of initial generation in Fig. 12a-c wherein the best location of initial generations is presented. From generation to generation, the number of the global Pareto front solutions increases sharply. The global optimal searching ability of the ENSES approach is clearly demonstrated. Through the iterations, the improvement in the solutions becomes smaller and smaller ultimately reaching a convergence as shown in Fig. 13. 1000 generations are made in order to get the convergence of welded sandwich beam with respect to the aforementioned constraints. Through the verification, it is found that the 500 solutions on the Pareto front are sufficient to derive the best solution with respect to the number of solutions and generations, i.e., the overall number of function evaluations.

However, providing more samples will lead to more effort in computational analysis. Thus, this issue reflects to uneconomical in computational cost even though a better result is obtained. Since ENSES is dealt with the component of ' $\mu+\lambda$ ', thus the convergence time is depending on the generations and populations generated during the analysis. The points of Pareto-optimal solution generated in the convergence stage are similar to the user-defined input value for generation's column.

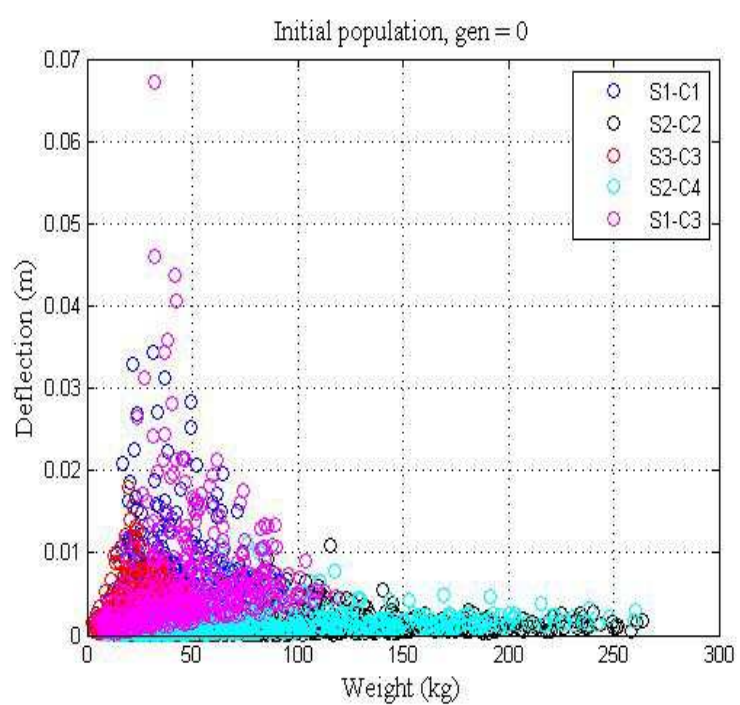

(a)

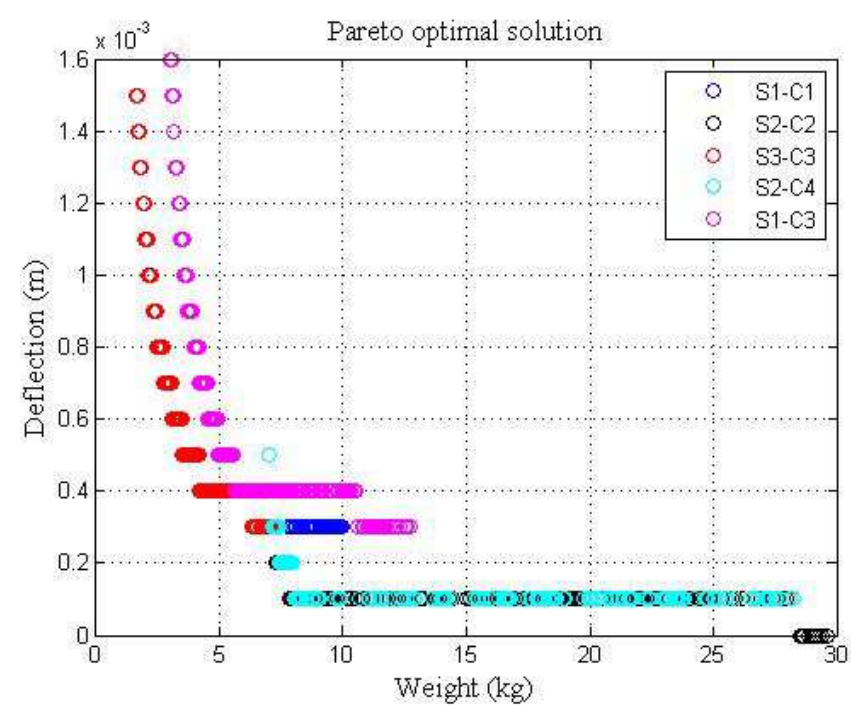

(b)

Fig. 11. Combinatorial in material properties of core and facing skins. Refer to Table 2 for the material configurations (a) Initial generation with combinatorial material properties (b) Pareto-optimal solutions with combinatorial material properties 


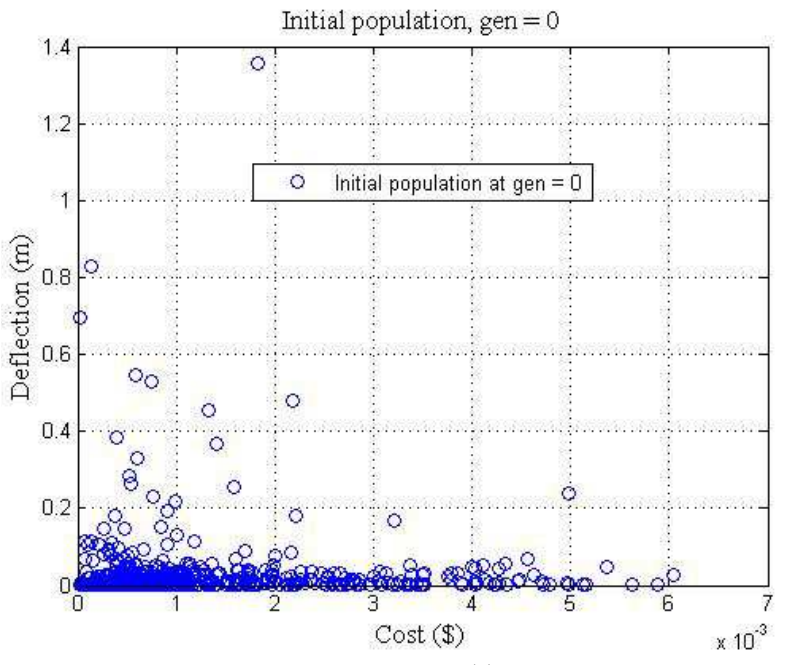

(a)

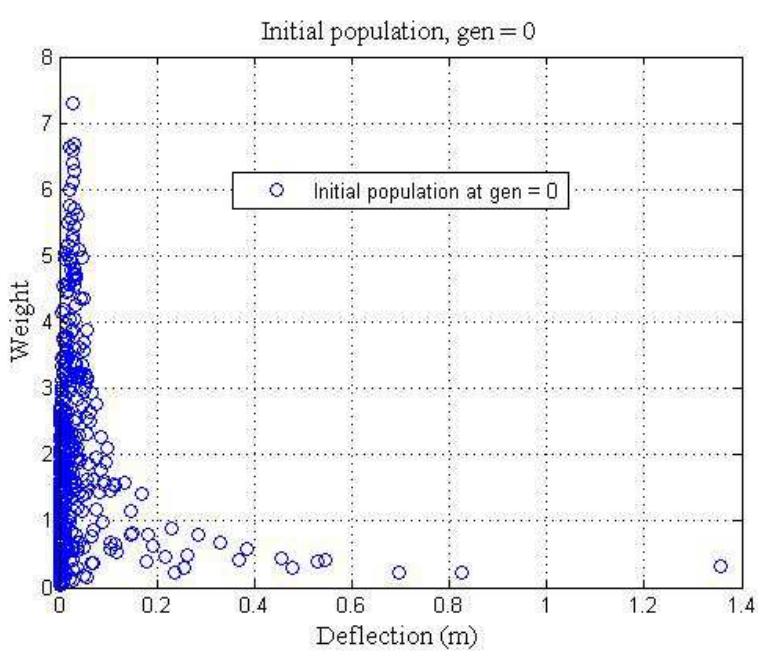

(c)

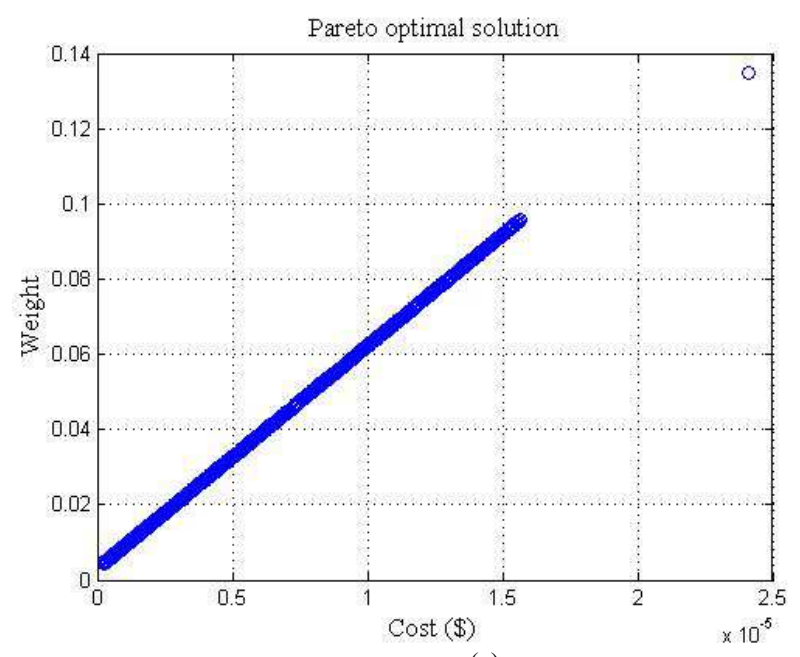

(e)

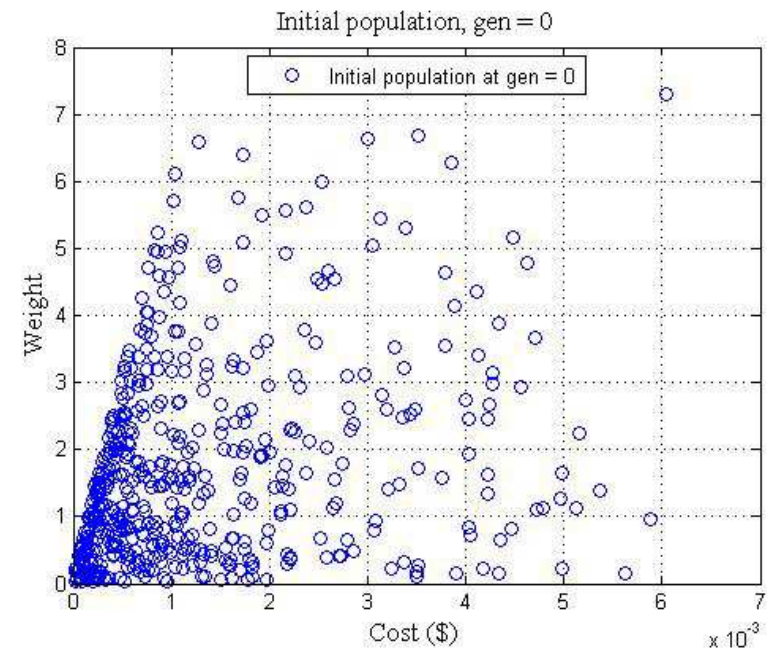

(b)

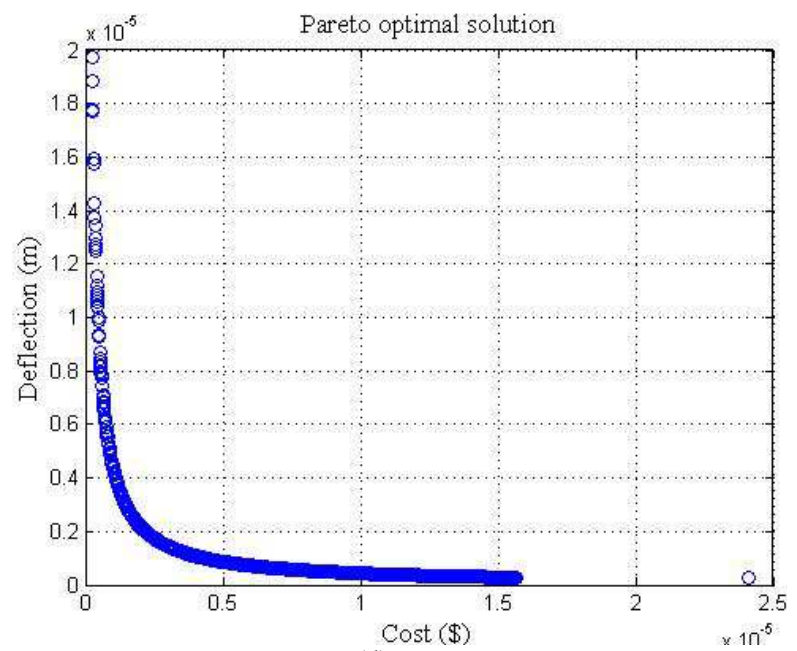

(d)

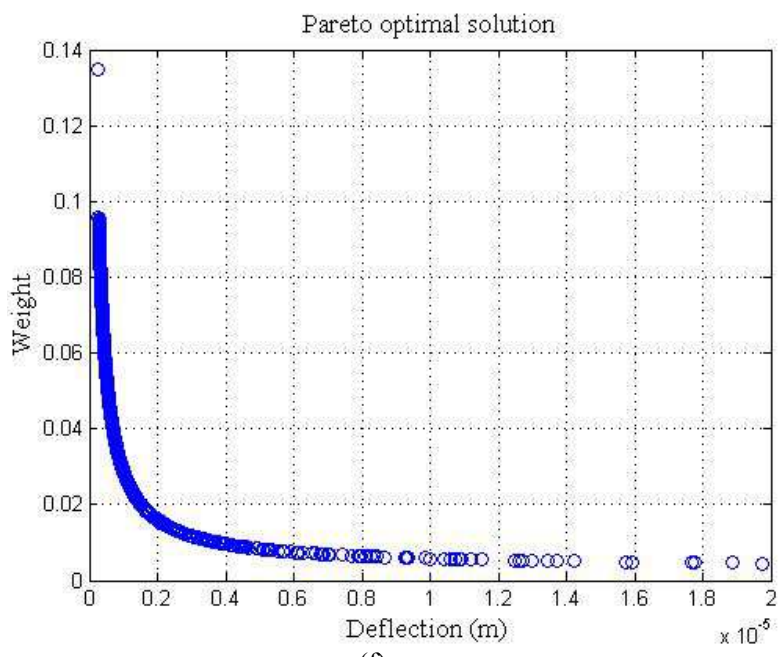

(f)

Fig. 12. Initial generation and Pareto-optimal solutions in optimizing the three objective functions of welded sandwich beam 


\section{Conclusion}

It has been proven for various combinatorial optimization problems that they can be solved by evolutionary algorithms in reasonable time using a suitable representation together with mutation operators adjusted to the given problem. A comprehensive of elitism approach is presented in order to find the solutions of global optima for two and three optimization problems. In this study, the ENSES model is built to integrate a variety of optimization objectives using ES as EA in the NSGA-II procedure, which involves an initialization, crossover and mutation operators. We have demonstrated and verified the model by applying it to two optimization problems. Case 1 has two nonlinear objective functions with respect to five nonlinear inequality constraints whilst Case 2 has three nonlinear objective functions with respect to three nonlinear and one linear inequality constraints. The input variables for Case 1 are the length $l_{s}$, the core $t_{c}$ and skin thicknesses $t_{f}$. For Case 2, four variables have been identified, which consists of the beam depth $t$, the beam width $b$, the weld length $l$ and the weld thickness $h$.

ENSES is found to be efficient due to its strategies like fitness sharing and solution diversity preservation. Eventually, the adoption of the ENSES models to a relatively well-defined beam problem have not only verified the robustness of the ENSES models, but also demonstrated the capability of the models to deal with more objectives and more variables. Though, the ENSES model only works for minimization problems. It is trivial to make a minimization problem $-f(x)$ from a maximization problem $f(x)$. Our evaluation outcomes illustrate that the model is useful as a support tool to optimize the beam design problems and other test functions. The potential of ENSES to cater more objectives and variables is very transparent due to its open-ended format even though we only tested them with two and three objective functions. Nevertheless, the current approach can be further improved in its efficiency and effectiveness. It also has to be mentioned that in certain situations, e.g., when preference information is included in the fitness assignment process and the preferences change over time, elitism may have its drawbacks. A comparison with other EMOAs such as SMS-EMOA or SPEA etc. instead of NSGA-II will be useful to the users in order to show the good performance with the similar test adaptations. In this context, the suggested algorithms could be useful to compare the models quantitatively and allowing a more accurate assessment towards the optimization problems.

Conclusively, a simple basis is presented in order to help the designers and users on the understanding of the model approach as well as the sandwich structure itself.
The design, weight and environmental benefits make the sandwich structures are always be the chosen materials in the constructions nowadays.

\section{Acknowledgement}

The authors gratefully acknowledge Ministry of Higher Education (Malaysia) for their financial supports and the opportunity given in completing this study. Special thanks to the reviewers for their commitment in reviewing the paper.

\section{Author's Contributions}

A.B. Ilyani Akmar: Designed and implemented the simulation model, analyzed and described the analytical model, carried out the stochastic implementation of the simulation and prepared the manuscript.

T. Rabczuk: Supervised the projects, giving ideas on the framework, commented on studied subject matter and edited the manuscript.

O. Kramer: Consulted on the stochastic implementation, giving ideas on the framework and edited the manuscript.

\section{Ethics}

The author(s) warrant that the manuscript submitted is their own original work. All authors participated in this study will take public responsibility for the work and have approved the manuscript. The manuscript has not been published or accepted for publication elsewhere, either in whole or in part, whether in English or another language. The manuscript meet ethical standards applicable to the research discipline and does not violate any intellectual property right of any person.

\section{References}

Amuso, V.J. and J. Enslin, 2007. The Strength Pareto Evolutionary Algorithm 2 (SPEA2) applied to simultaneous multi-mission waveform design. Proceedings of the International Waveform Diversity and Design Conference, Jun. 4-8, IEEE Xplore Press, Pisa, pp: 407-417. DOI: 10.1109/WDDC.2007.4339452

Ashby, M., 2011. Hybrid materials to expand the boundaries of material-property space. J. Am. Ceramic Society, 94: s3-s14. DOI: $10.1111 /$ j.1551-2916.2011.04559.x

Bannister, M.K., R. Braemar and P.J. Crothers, 1999. The mechanical performance of 3D woven sandwich composites. Composite Structures, 47: 687-690. DOI: $10.1016 / \mathrm{S} 0263-8223(00) 00035-0$ 
Beyer, H.G. and H.P. Schwefel, 2002. Evolution strategiesa comprehensive introduction. Natural Comput. Int. J., 1: 3-52. DOI: 10.1023/A:1015059928466

Can, W., C. Hao-ran and L. Zhen-kun, 2010. Experimental investigation of interfacial fracture behavior in foam core sandwich beams with visco-elastic adhesive interface. Composite Structures, 92: 1085-1091. DOI: 10.1016/j.compstruct.2009.10.011

Cao, K., M. Batty, B. Huang, Y. Liu and L. Yu et al., 2011. Spatial multi-objective land use optimization: Extensions to the non-dominated sorting genetic algorithm-II. Int. J. Geograph. Inform. Sci., 25: 1949-1969. DOI: 10.1080/13658816.2011.570269

Chen, G., X. Han, G. Liu, C. Jiang and Z. Zhao, 2012. An efficient multi-objective optimization method for black-box functions using sequential approximate technique. Applied Soft Comput., 12: 14-27. DOI: 10.1016/j.asoc.2011.09.011

Coello, C.A.C., 1999. An updated survey of evolutionary multiobjective optimization techniques: State of the art and future trends. Proceedings of the Congress on Evolutionary Computation, Jul. 6-9, IEEE Xplore Press, Washington, DC.

DOI: $10.1109 /$ CEC.1999.781901

Composites, H., 2000. HexWeb ${ }^{\mathrm{TM}}$ honeycomb sandwich design technology. Hexcel Composites.

Corne, D.W., N.R. Jerram, J.D. Knowles and M.J. Oates, 2001. PESA-II: Region-based selection in evolutionary multiobjective optimization. Proceedings of the Genetic and Evolutionary Computation Conference, (ECC' 01), pp: 283-290.

Deb, K. and T. Goel, 2001. Controlled elitist nondominated sorting genetic algorithms for better convergence. Proceedings of the 1st International Conference on Evolutionary Multi-Criterion Optimization, Mar. 7-9, Springer Berlin Heidelberg, Zurich, Switzerland, pp: 67-81. DOI: $10.1007 / 3-540-44719-95$

Deb, K., 2001. Multi-Objective Optimization Using Evolutionary Algorithms. 1st Edn., John Wiley and Sons, Chichester, ISBN-10: 047187339X, pp: 497.

Deb, K., A. Pratap, S. Agarwal and T. Meyarivan, 2002. A fast and elitist multiobjective genetic algorithm: NSGA-II. IEEE Trans. Evolut. Comput., 6: 182-197. DOI: $10.1109 / 4235.996017$

DIAB, 2012. Sandwich composites and core materials: How they work and why you should use them. DIAB Group.

Eiben, A.E. and J.E. Smith, 2003. Introduction to Evolutionary Computing. 1st Edn., Springer Science and Business Media, Berlin, ISBN-10: 3540401849 , pp: 299.
Fonseca, C.M. and P.J. Fleming, 1993. Genetic algorithms for multiobjective optimization: Formulationdiscussion and generalization. Proceedings of the 5th International Conference on Genetic Algorithms, (CGA' 93), Morgan Kaufmann Publishers Inc., San Francisco, pp: 416-423.

Garcia, G.A.O., 2011. Elitist Non-dominated Sorting Evolution Strategy (ENSES).

Goldberg, D.E., 1989. Genetic Algorithms in Search, Optimization and Machine Learning. 1st Edn., Addison-Wesley, Reading, ISBN-10: 0201157675, pp: 412.

Guilleminot, J., S. Comas-Cardona, D. Kondo, C. Binetruy and P. Krawczak, 2008. Multiscale modelling of the composite reinforced foam core of a 3D sandwich structure. Composites Sci. Technol., 68: 1777-1786.

DOI: 10.1016/j.compscitech.2008.02.005

Hajela, P. and C.Y. Lin, 1992. Genetic search strategies in multicriterion optimal design. Structural Optimiz., 4: 99-107. DOI: 10.1007/BF01759923

Horn, J., N. Nafpliotis and D.E. Goldberg, 1994. A niched pareto genetic algorithm for multiobjective optimization. Proceedings of the First IEEE Conference on Evolutionary Computation, Jun. 37-39, IEEE Xplore Press, Orlando, FL., pp: 82-87. DOI: 10.1109/ICEC.1994.350037

Hussain, T.S., 1998. An introduction to evolutionary computation. Queen's University.

Kursawe, F., 1991. A variant of evolution strategies for vector optimization. Proceedings of the 1st Workshop on Parallel Problem Solving from Nature, Oct. 1-3, Springer, pp: 193-197. DOI: 10.1007/BFb0029752

Lascoup, B., Z. Aboura, K. Khellil and M. Benzeggagh, 2010. Homogenization of the core layer in stitched sandwich structures. Composites Sci. Technol., 70: 350-355. DOI: 10.1016/j.compscitech.2009.11.006

Liu, B., F.V. Fernandez, Q. Zhang, M. Pak and S. Sipahi et al., 2010. An enhanced MOEA/D-DE and its application to multiobjective analog cell sizing. Proceedings of the IEEE Congress on Evolutionary Computation, Jul. 18-23, IEEE Xplore Press, Barcelona, pp: 1-7. DOI: 10.1109/CEC.2010.5585957

Mori, L.F., S. Lee, Z.Y. Xue, A. Vaziri and D.T. Queheillalt et al., 2007. Deformation and fracture modes of sandwich structures subjected to underwater impulsive loads. J. Mechan. Materials Structures, 2: 1981-2006. DOI: 10.2140/jomms.2007.2.1981

Mosavi, A. and A. Vaezipour, 2012. Reactive search optimization; application to multiobjective optimization problems. Applied Mathemat., 3: 1572-1582. DOI: 10.4236/am.2012.330217 
Nebro, A.J., F. Luna, E. Alba, B. Dorronsoro and J.J. Durillo et al., 2008. AbYSS: Adapting scatter search to multiobjective optimization. IEEE Trans. Evolut. Comput., 12: 439-457. DOI: $10.1109 /$ TEVC.2007.913109

Osyczka, A. and S. Kundu, 1995. A new method to solve generalized multicriteria optimization problems using the simple genetic algorithm. Structural Optimiz., 10: 94-99. DOI: 10.1007/BF01743536

Rabczuk, T., J.Y. Kim, E. Samaniego and T. Belytschko, 2004. Homogenization of sandwich structures. Int. J. Numerical Methods Eng., 61: 1009-1027. DOI: $10.1002 /$ nme. 1100

Rangavajhala, S., A.A. Mullur and A. Messac, 2006. Uncertainty visualization in multiobjective robust design optimization. Proceedings of the 47th AIAA/ASME/ASCE/ AHS Structures, Structural Dynamics and Materials Conference, May 1-4, Newport. Rhode Island, pp: 1-16. DOI: $10.2514 / 6.2006-1813$

Ratwani, M.M., 2010. Composite materials and sandwich structures: A Primer.

Rechenberg, I., 1973. Evolutionsstrategie: Optimierung Technischer Systeme Nach Prinzipien der Biologischen Evolution. 1st Edn., FrommannHolzboog, ISBN-10: 3772803733, pp: 170.

Schaffer, J.D., 1985. Some experiments in machine learning using vector evaluated genetic algorithms. $\mathrm{PhD}$ Thesis, Vanderbilt Univ.,Nashville, TN.

Schwefel, H.P., 1981. Numerical Optimization of Computer Models. 1st Edn., John Wiley and Sons, ISBN-10: 0471099880, pp: 398.

Seshadri, A., 2011. A fast elitist multi-objective genetic algorithm: NSGA-II.

Spears, W.M., K.A. DeJong, T. Back, D.B. Fogel and H. de Garis, 1993. An overview of evolutionary computation. Proceedings of the European Conference on Machine Learning, Apr. 5-7, Springer Berlin Heidelberg, Austria, pp: 442-459. DOI: $10.1007 / 3-540-56602-3 \_163$
Srinivas, N. and K. Deb, 1994. Muiltiobjective optimization using nondominated sorting in genetic algorithms. Evolut. Comput., 2: 221-248.

DOI: $10.1162 /$ evco.1994.2.3.221

Steeves, C.A. and N. A. Fleck. 2004a. Material selection in sandwich beam construction. Scripta Mater., 50: 1335-1339. DOI: 10.1016/j.scriptamat.2004.02.015

Steeves, C.A. and N.A. Fleck, 2004b. Collapse mechanisms of sandwich beams with composite faces and a foam core, loaded in three-point bending. Part I: Analytical models and minimum weight design. Int. J. Mechanical Sci., 46: 561-583. DOI: 10.1016/j.ijmecsci.2004.04.003

Tan, K.C., T.H. Lee and E.F. Khor, 2001. Evolutionary algorithms for multi-objective optimization: Performance assessments and comparisons. Proceedings of the IEEE Congress on Evolutionary Computation, May 27-30, IEEE Xplore Press, Seoul, pp: 979-986. DOI: 10.1109/CEC.2001.934296

Xia, F. and X.Q. Wu, 2010. Work on impact properties of foam sandwich composites with different structure. J. Sandwich Structures Mater., 12: 47-62. DOI: $10.1177 / 1099636209106256$

Zitzler, E., 1999. Evolutionary algorithms for multiobjective optimization: Methods and applications. Shaker Ithaca.

Zitzler, E., K. Deb and L. Thiele, 2000. Comparison of multiobjective evolutionary algorithms: Empirical results. Evolut. Comput., 8: 173-195. DOI: $10.1162 / 106365600568202$

Zitzler, E., M. Laumanns and L. Thiele, 2001. SPEA2: Improving the strength pareto evolutionary algorithm for multiobjective optimization. CIMNE, Barcelona, Spain. 